\title{
Bose-Einstein condensation under external conditions
}

\author{
Klaus Kirsten [团] \\ University of Leipzig \\ Institute of Theoretical Physics \\ Augustusplatz 10, 04109 Leipzig \\ and \\ David J. Toms [闻] \\ Department of Physics \\ University of Newcastle Upon Tyne, \\ Newcastle Upon Tyne, U. K. NE1 7RU \\ July 7,2021
}

\begin{abstract}
We discuss the phenomenon of Bose-Einstein condensation under general external conditions using connections between partition sums and the heat-equation. Thermodynamical quantities like the critical temperature are given in terms of the heat-kernel coefficients of the associated Schrödinger equation. The general approach is applied to situations where the gas is confined by arbitrary potentials or by boxes of arbitrary shape.
\end{abstract}


One of the most striking developments of the last few years has been the experimental observation of Bose-Einstein condensation in very cold gases of rubidium [1], lithium [2] and sodium [3]. The systems are very dilute and as a first approximation would be expected to be well described by a boson gas model with no interactions among the atoms. The atoms are confined in complicated magnetic traps which can be modeled by harmonic oscillator potentials. Nowadays one can say that the thermodynamic properties of the confined gas are very well understood, including the effects that the finite number of particles has on the system «, 5, 6, 7, 8, 9] as well as the influence of a weak interaction among the atoms [10]. The above mentioned results have all been obtained in the grand canonical approach, which, although inadequate for the calculation of, for example, flucuations of particle numbers 11, 12, 13, 14 is still reliable for thermodynamical properties such as the critical temperature or the ground state occupation number.

Although recently most interest is in Bose-Einstein condensation in magnetic traps also different confining potentials [15] or gases confined inside a rectangular box [16] have been considered and specific differences have been stated. A formal discussion of boundary effectis has been given in [17]. All this research may be called analysis of Bose-Einstein condensation under external conditions.

In this article we want to provide an approach which enables one to deal with any kind of external conditions (including the most relevant anisotropic harmonic oscillator potential). The methods we are going to employ are the so-called heat-kernel techniques extensively used in finite temperature relativistic quantum field theory starting with the work of Dowker and Kennedy [18]. (See also [19].) In non-relativistic theories these techniques are however nearly unemployed and we want to show in the present article that they can also be used here in a very effective way.

Let us consider a system of $N$ non-interacting bosons under external conditions such that the single particle state energies $E_{N}$ are determined by the Schrödinger equation

$$
-\frac{\hbar^{2}}{2 m} \Delta \phi_{N}(x)+V(x) \phi_{N}(x)=E_{N} \phi_{N}(x),
$$

where $V(x)$ is the confining potential. For example in [15] the confining potential

$$
V(x)=\sum_{i=1}^{d} \epsilon_{i}^{p_{i}}\left|x_{i}\right|^{p_{i}}
$$


has been considered in the thermodynamic limit. If in addition the gas is confined by a box $\mathcal{M}$ one has to impose suitable boundary conditions at the boundary $\partial \mathcal{M}$ of the box. Without specifying the spectrum $E_{N}$ (as a rule it will not be known explicitly) the grand canonical potential may be given in the form

$$
q=q_{0}+\sum_{n=1}^{\infty} \sum_{N}^{\prime} \frac{1}{n} e^{-\beta n\left(E_{N}-\mu\right)} .
$$

Here

$$
q_{0}=-d_{0} \ln \left(1-z e^{-\beta E_{0}}\right),
$$

is the contribution of the ground state with energy $E_{0}, d_{0}$ is its degeneracy and $z=\exp (\beta \mu)$ is the fugacity. The prime in (3) indicates that the ground state contribution is to be omitted from the sum.

For the evaluation of this kind of expression it is very effective to make use of the Mellin-Barnes integral representation [20]

$$
e^{-v}=\frac{1}{2 \pi i} \int_{c-i \infty}^{c+i \infty} d \alpha \Gamma(\alpha) v^{-\alpha}
$$

valid for $\Re v>0$ and $c \in \mathbb{R}, c>0$. This is easily proven by closing the contour to the left obtaining the power series expansion of $\exp (-v)$. As an immediate consequence of eqs. (3) and (4) the grand canonical partition sum may be casted into the form

$$
q=q_{0}+\frac{1}{2 \pi i} \int_{c-i \infty}^{c+i \infty} d \alpha \Gamma(\alpha) \beta^{-\alpha} L i_{1+\alpha}\left(e^{-\beta\left(\mu_{c}-\mu\right)}\right) \zeta(\alpha)
$$

with the polylogarithm

$$
\operatorname{Li}_{n}(x)=\sum_{l=1}^{\infty} \frac{x^{l}}{l^{n}}
$$

and the spectral zeta function

$$
\zeta(\alpha)=\sum_{N}^{\prime}\left(E_{N}-E_{0}\right)^{-\alpha}
$$


Here we introduced the variable $\mu_{c}$ which denotes the value of the chemical potential at the transition temperature. For ideal gases this value is identical with the ground state energy, $\mu_{c}=E_{0}$. The parameter $c$ in eq. (5) is given in a way such that all poles of the integrand lie to the left of the contour. Closing the contour to the left one picks up the right-most residues of $\zeta(\alpha)$. Concerning their determination there are deep connections between the zeta function of an operator and its heat-kernel defined as

$$
K(t)=\sum_{N}^{\prime} e^{-t\left(E_{N}-E_{0}\right)}
$$

The small-t behaviour

$$
K(t) \sim \sum_{k=0}^{\infty} b_{k} t^{-j_{k}}
$$

is of particular relevance here. $b_{k}$ are the so-called heat-kernel coefficients. We assume $j_{k}>j_{k+1}$, and the connection between the residues of $\zeta(\alpha)$, which occur at $\alpha=j_{k}$, and the heat-kernel coefficients reads

$$
\operatorname{Res} \zeta\left(\alpha=j_{k}\right)=\frac{b_{k}}{\Gamma\left(j_{k}\right)}
$$

Taking into consideration only the two right most poles of $\zeta(\alpha)$ one arrives at

$$
q=q_{0}+\beta^{-j_{0}} L i_{1+j_{0}}\left(e^{-\beta\left(\mu_{c}-\mu\right)}\right) b_{0}+\beta^{-j_{1}} L i_{1+j_{1}}\left(e^{-\beta\left(\mu_{c}-\mu\right)}\right) b_{1}+\ldots
$$

For the determination of the critical temperature and the thermodynamical properties of the gas up to $T_{c}$ the above approximation can be shown to be a very good one.

We thus have a representation of the grand canonical partition sum in terms of the heat-kernel coefficients of the relevant Schrödinger operator. What have we gained with this representation? This becomes clear by noticing that the heat-kernel coeficients $b_{0}, b_{1}$ are known or easily determined for a very large class of Schrödinger operators including the situation where the gas is confined by arbitrary potentials or boxes of arbitrary shape. This includes physical situations which are of relevance in recent experiments and also those which might be of relevance in future ones. 
Let us exemplify the power of the approach by considering further the particle number and the critical temperature. The particle number is

$$
\begin{aligned}
N & =\left.\beta^{-1}\left(\frac{\partial q}{\partial \mu}\right)\right|_{T, V} \\
& =N_{0}+\beta^{-j_{0}} L i_{j_{0}}\left(e^{-\beta\left(\mu_{c}-\mu\right)}\right) b_{0}+\beta^{-j_{1}} L i_{j_{1}}\left(e^{-\beta\left(\mu_{c}-\mu\right)}\right) b_{1}+\ldots
\end{aligned}
$$

In order to avoid distinguishing all the different cases, let us assume from now on that $j_{1}>1$. In this case, the critical temperature $1 / \beta_{c}$ is determined by

$$
N=\beta_{c}^{-j_{0}} \zeta_{R}\left(j_{0}\right) b_{0}+\beta_{c}^{-j_{1}} \zeta_{R}\left(j_{1}\right) b_{1}+\ldots,
$$

and reads

$$
T_{c}=T_{0}\left\{1-\frac{\zeta_{R}\left(j_{1}\right) b_{1}}{j_{0} \zeta_{R}\left(j_{0}\right)^{j_{1} / j_{0}} b_{0}^{j_{1} / j_{0}}} \frac{1}{N^{\left(j_{0}-j_{1}\right) / j_{0}}}\right\}
$$

with

$$
T_{0}=\frac{1}{k}\left(\frac{N}{\zeta_{R}\left(j_{0}\right) b_{0}}\right)^{1 / j_{0}}
$$

the critical temperature in the bulk limit. Let us stress that eq. (14) contains the influence that the finite number $N$ of particles have, the details being encoded in the exponents $j_{i}$ and the coefficients $b_{i}$. Proceeding with the energy and the specific heat, all thermodynamical quantities are easily displayed in terms of the heat-kernel coefficients. If $j_{1} \leq 1$ different results are found due to the behaviour of the polylogarithm [21].

The presented technique allows us to calculate thermodynamical properties by treating the sums over the discrete energy levels. Another possible way to do this analysis is to approximate the sums by integrals. A crucial feature in obtaining a reliable approximation is to use an appropriate density of states [5]. By using a refinement of Karamatas' theorem [22], one can show that the use of the density

$$
\rho(E)=\frac{b_{0}}{\Gamma\left(j_{0}\right)} E^{j_{0}-1}+\frac{b_{1}}{\Gamma\left(j_{1}\right)} E^{j_{1}-1}
$$

is completely equivalent to the analysis presented above. 
A slightly different approach results by the use of the effective fugacity (for the anisotropic harmonic oscillator see [7])

$$
z_{e f f}=z e^{-\beta E_{1}}
$$

where $E_{1}$ is the first excited level with, lets say, degeneracy $d_{1}$. Whereas in the previous calculation only the groundstate has been treated separately, we now separate the groundstate and the first excited level to find

$$
q=q_{0}+d_{1} L i_{1}\left(z_{e f f}\right)+\beta^{-j_{0}} L i_{1+j_{0}}\left(z_{e f f}\right) b_{0}+\beta^{-j_{1}} L i_{1+j_{1}}\left(z_{e f f}\right) b_{1}+\ldots
$$

This expansion is a very good approximation even below the critical temperature (for the harmonic oscillator see [7]). Also here thermodynamical properties are obtained in the same manner as before.

Let us now use our general results to consider the special situations mentioned already several times.

Example 1: Gas inside a $d$-dimensional box of arbitrary shape.

In the notation of equation (11) the situation is described by $V(x)=0$ and $\left.\phi_{N}(x)\right|_{x \in \partial \mathcal{M}}=0$. (Other boundary conditions can be treated as well.) For this example the relevant heat-kernel coefficients are known (see f.e. [23]) and read

$$
\begin{aligned}
& b_{0}=(4 \pi)^{-d / 2}\left(2 m / \hbar^{2}\right)^{d / 2} \operatorname{vol}(\mathcal{M}) \\
& b_{1}=-(4 \pi)^{-d / 2}\left(2 m / \hbar^{2}\right)^{(d-1) / 2}(\sqrt{\pi} / 2) \operatorname{vol}(\partial \mathcal{M})
\end{aligned}
$$

together with $j_{0}=d / 2, j_{1}=(d-1) / 2$. Here $\operatorname{vol}(\mathcal{M})$ and $\operatorname{vol}(\partial \mathcal{M})$ denote the finite volume of the box and its boundary respectively. For the critical temperature this means

$$
T_{c}=T_{0}\left\{1+\frac{1}{2 d} \frac{\operatorname{vol}(\partial \mathcal{M})}{(\operatorname{vol}(\mathcal{M}))^{(d-1) / d}} \frac{\zeta_{R}((d-1) / 2)}{\zeta_{R}(d / 2)^{(d-1) / d}} \frac{1}{N^{1 / d}}\right\}
$$

where

$$
T_{0}=\frac{h^{2}}{2 \pi m k}\left(\frac{N}{\operatorname{vol}(\mathcal{M}) \zeta_{R}(d / 2)}\right)^{2 / d} .
$$

The rectangular three dimensional box has been analysed in detail in [16] using a density of states approach. 
Example 2: $d$-dimensional isotropic harmonic oscillator as confining potential.

Let us now choose the confining potential

$$
V(x)=\frac{1}{2} \hbar \omega m \sum_{i=1}^{d} x_{i}^{2}
$$

In this case the coefficients are found by just calculating with geometric series [6]. The result is $b_{0}=(\hbar \omega)^{-d}, b_{1}=(\hbar d \omega / 2) b_{0}$ and $j_{0}=d, j_{1}=d-1$ and so

$$
T_{c}=T_{0}\left\{1-\frac{\zeta_{R}(d-1)}{2 \zeta_{R}(d)^{(d-1) / d}} \frac{1}{N^{1 / d}}\right\}
$$

with

$$
T_{0}=\hbar \omega\left(\frac{N}{\zeta_{R}(d)}\right)^{1 / d}
$$

(see also f.e. [24]).

Example 3: 3-dimensional anisotropic harmonic osciallator as confining potential.

In the anisotropic case the relevant potential reads

$$
V(x)=\frac{1}{2} \hbar m\left(\omega_{1} x_{1}^{2}+\omega_{2} x_{2}^{2}+\omega_{3} x_{3}^{2}\right)
$$

With the same comments as above one gets here $j_{0}=3, j_{1}=2$, and

$$
b_{0}=\frac{1}{\hbar^{3} \omega_{1} \omega_{2} \omega_{3}} ; \quad b_{1}=\frac{1}{2 \hbar^{2}}\left(\frac{1}{\omega_{1} \omega_{2}}+\frac{1}{\omega_{1} \omega_{3}}+\frac{1}{\omega_{2} \omega_{3}}\right)
$$

For the critical temperature this means

$$
T_{c}=T_{0}\left\{1-\frac{\zeta_{R}(2)}{3 \zeta_{R}(3)^{2 / 3}} \gamma N^{-1 / 3}\right\}
$$

with

$$
\gamma=\frac{1}{2}\left(\omega_{1} \omega_{2} \omega_{3}\right)^{2 / 3}\left[\frac{1}{\omega_{1} \omega_{2}}+\frac{1}{\omega_{1} \omega_{3}}+\frac{1}{\omega_{2} \omega_{3}}\right]
$$

and

$$
T_{0}=\hbar\left(\omega_{1} \omega_{2} \omega_{3}\right)^{1 / 3}\left(\frac{N}{\zeta_{R}(3)}\right)^{1 / 3}
$$


These results have also been obtained using an approach based on the EulerMaclaurin formula [7] and based on a density of states approach [5].

Analogous formulas for the $d$-dimensional anisotropic harmonic osciallator can be found in an easy manner.

Example 4: Arbitrary power law confining potential in 3 dimensions.

Let us finally consider the potential

$$
V(x)=\epsilon^{p} \sum_{i=1}^{3}\left|x_{i}\right|^{p}
$$

where in order to simplify comparison with the harmonic oscillator calculation we use the notation

$$
\epsilon=\sqrt{\frac{m}{2}} \hbar^{\frac{2-p}{2 p}} \omega^{\frac{p+2}{2 p}}
$$

Using the results of [25] for the resumed heat-kernel, one gets for this case

$$
b_{0}=\frac{8 \Gamma^{3}(1 / p)}{\pi^{3 / 2} p^{3}} \frac{1}{(\hbar \omega)^{\frac{3}{2}(1+2 / p)}}
$$

and $b_{1}=E_{0} b_{0}$, together with $j_{0}=\frac{3}{2}+\frac{3}{p}$ and $j_{1}=j_{0}-1$. The resulting critical temperature for this example is then easily found to be

$$
T_{c}=T_{0}\left\{1-\frac{\left(E_{0} / \hbar \omega\right) \zeta_{R}(1 / 2+3 / p)}{(3 / 2+3 / p) \zeta_{R}(3 / 2+3 / p)^{\frac{p+6}{3(p+2)}}}\left(\frac{2 \Gamma(1 / p)}{\sqrt{\pi} p}\right)^{\frac{2 p}{p+2}} \frac{1}{N^{\frac{2 p}{3(p+2)}}}\right\} .
$$

The bulk critical temperature here is

$$
T_{0}=\hbar \omega\left(\frac{\sqrt{\pi} p}{2 \Gamma(1 / p)}\right)^{\frac{2 p}{p+2}}\left(\frac{N}{\zeta_{R}(3 / 2+3 / p)}\right)^{\frac{2 p}{3(p+2)}}
$$

(for further bulk properties see [15]).

In summary we have shown, that our approach enables us to deal with complicated external conditions in an efficient way. Of course we do not claim that the results presented in the examples cannot be obtained by other means; in fact, most of the results for $T_{c}$ have been obtained differently. However, the unified treatment of many different situations seems very elegant to us. Having at hand this general approach given an ideal Bose gas under arbitrary external conditions one only has to determine the leading heat-kernel 
coefficients of the associated Schrödinger operator (or even only take them from the literature) in order to know the whole thermodynamical behaviour up to or below the critical temperature. Without technical complications our approach includes the effect of a finite number of particles.

These ideas are also applicable to the canonical ensemble. Furthermore, due to the connections between the microcanonical and the grand canonical ensemble 13] it seems possible to develop this approach also for the microcanonical ensemble.

Acknowledgements This investigation has been partly supported by the DFG under contract number BO1112/4-2.

\section{References}

[†] kirsten@tph100.physik.uni-leipzig.de.

[†] d.j.toms@newcastle.ac.uk.

[1] M. H. Anderson, J. R. Ensher, M. R. Matthews, C. E. Wieman, and E. A. Cornell, Science 269 (1995) 198.

[2] C. C. Bradley, C. A. Sackett, J. J. Tollett, and R. G. Hulet, Phys. Rev. Lett. 75 (1995) 1687.

[3] K. B. Davis, M.-O. Mewes, M. R. Andrews, N. J. van Druten, D. S. Durfee, D. M. Kurn, and W. Ketterle, Phys. Rev. Lett. 75 (1995) 3969.

[4] W. Ketterle and N.J. van Druten, Phys. Rev. A 54 (1996) 656.

[5] S. Grossmann and M. Holthaus, Phys. Lett. A 208 (1995) 188. S. Grossmann and M. Holthaus, Zeit. für Naturforschung 50 a (1995) 921.

[6] K. Kirsten and D. J. Toms, Phys. Rev. A 54 (1996) 4188.

K. Kirsten and D. J. Toms, Phys. Lett. A 222 (1996) 148.

[7] H. Haugerud, T. Haugset and F. Ravndal, Phys. Lett. A 225 (1997) 18. T. Haugset, H. Haugerud and J.O. Anderson, Phys. Rev. A 55 (1997) 2922. 
[8] F. Brosens, J. T. Devreese and L. F. Lemmens, Solid St. Comm. 100 (1996) 123.

F. Brosens, J. T. Devreese and L. F. Lemmens, Phys. Rev. E 55 (1997) 227.

F. Brosens, J. T. Devreese and L. F. Lemmens, Phys. Rev. E 55 (1997) 6795.

[9] G.-L. Ingold and A. Lambrecht, Eur. Phys. J. D 1 (1998) 29.

[10] S. Giorgini, L.P. Pitaevskii and S. Stringari, Phys. Rev. A 54 (1996) 4633.

S. Giorgini, L.P. Pitaevskii and S. Stringari, Phys. Rev. Lett. 78 (1997) 3987.

S. Giorgini, L.P. Pitaevskii and S. Stringari, J. Low. Temp. Phys. 109 (1997) 309 .

[11] P. Navez, D. Bitouk, M. Gajda, Z. Idziaszek and K. Rzazewski, Phys. Rev. Lett. 79 (1997) 1789.

M. Gajda and K. Rzazewski, Phys. Rev. Lett. 78 (1997) 2686.

[12] S. Grossmann and M. Holthaus, Phys. Rev. Lett. 79 (1997) 3557.

[13] S. Grossmann and M. Holthaus, Optics Express 1 (1997) 262.

[14] M. Wilkens and C. Weiss, "Universality classes and particle number fluctuations of trapped ideal Bose gases" (Preprint, Potsdam, 1997).

[15] V. Bagnato, D. E. Pritchard, and D. Kleppner, Phys. Rev. A 35 (1987) 4354.

[16] S. Grossmann and M. Holthaus, Z. Phys. B 97 (1995) 319.

[17] R. M. Ziff, G. E. Uhlenbeck and M. Kac. Phys. Rep. 32 (1977) 169.

[18] J. S. Dowker and G. Kennedy, J. Phys. A 11 (1978) 895.

[19] J. S. Dowker and J. P. Schofield, Nucl. Phys. B 327 (1989)267.

K. Kirsten, J. Phys. A 24 (1991)3281.

K. Kirsten, Class. Quantum Grav. 8 (1991) 2239.

D. J. Toms, Phys. Rev. Lett. 8 (1992) 1152.

D. J. Toms, Phys. Rev. D 47 (1993) 2483. 
[20] E. Elizalde, K. Kirsten and S. Zerbini, J. Phys. A: Math. Gen. 28 (1995) 617.

A.A. Bytsenko, L. Vanzo and S. Zerbini, Phys. Lett. B 291 (1992) 26.

[21] K. Kirsten and D. J. Toms, Bose-Einstein condensation in arbitrarily shaped cavities, in preparation.

[22] F. H. Brownell, Pacific J. Math. 5(1955) 483; J. Math. Mech. 6 (1957) 119.

H. P. Baltes and E. R. Hilf, Spectra of Finite Systems (Bibliographisches Institut Mannheim, Zurich, 1976).

[23] T. B. Branson and P. B. Gilkey, Commun. PDE 15 (1990) 245.

[24] W. J. Mullin, J. Low. Temp. Phys. 106 (1997) 615.

[25] L. Parker and D.J. Toms, Phys. Rev. D 31 (1985) 953.

L. Parker and D.J. Toms, Phys. Rev. D 31 (1985) 2424.

I. Jack and L. Parker, Phys. Rev. D 31 (1985) 2439. 